\title{
On the Detection of CE-OFDM Signals
}

\author{
João Guerreiro, Rui Dinis, and Paulo Montezuma
}

\begin{abstract}
In this letter, we study the optimum performance of constant envelope orthogonal frequency division multiplexing (CE-OFDM) signals in both ideal additive white Gaussian noise channels and frequency-selective channels, and we compare it to the performance of conventional CE-OFDM receivers based on a phase detector. It is shown that the phase detector can achieve optimum performance, but only in scenarios where the power efficiency is very low. For this reason, the use of CE-OFDM schemes in power-constrained scenarios may demand other type of receivers, such as optimum-based receivers.
\end{abstract}

Index Terms-OFDM, phase modulation, nonlinear distortion effects, optimum performance.

\section{INTRODUCTION}

$\mathbf{O}$ RTHOGONAL frequency division multiplexing (OFDM) schemes are widely employed in wireline and wireless communication systems. This is justified by their high robustness against multipath propagation, high spectral efficiency, flexibility and ease of implementation. However, in spite of the large number of techniques that have been proposed for reducing the envelope fluctuations of OFDM signals [1], the amplification problems of OFDM are still a severe drawback, and the use of highly efficient, strongly nonlinear power amplifiers remains difficult since they require signals with a quasi-constant envelope [2]. Recently, constant envelope OFDM (CE-OFDM) schemes were proposed to solve these amplification problems [3], [4]. The main idea behind CE-OFDM is to submit a conventional OFDM signal to a phase modulation process, which leads to a constant-envelope signal, suitable for strongly nonlinear power amplifiers, while most of the advantages inherent to OFDM are preserved. Clearly, the phase modulation is a nonlinear operation that introduces distortion effects in the transmitted signals leading to out-of-band radiation and a DC component that can substantially degrade the performance [5].

Conventionally, the detection of CE-OFDM signals involves a phase detector [3]. However, receivers based on a phase demodulation process are only suitable when the phase does not have excursions over $\pm \pi$, which can not always be

Manuscript received July 11, 2016; accepted July 27, 2016. Date of publication August 5, 2016; date of current version November 9, 2016. This work was partially supported by Instituto de Telecomunicações and Fundação para a Ciência e Tecnologia (FCT) under the project UID/EEA/50008/2013 IT (pluriannual founding) and the PhD grant SFRH/BD/90997/2012. The associate editor coordinating the review of this letter and approving it for publication was B. Smida.

J. Guerreiro and R. Dinis are with the Departamento de Engenharia Electrotecnica, Faculdade de Ciências e Tecnologia, Universidade Nova de Lisboa, 2829-516 Almada, Portugal, and also with IT, Instituto de Telecomunicações, 1049-001 Lisbon, Portugal (e-mail: jguerreiro.network@gmail.com; rdinis@fct.unl.pt).

P. Montezuma is with the Departamento de Engenharia Electrotecnica, Faculdade de Ciências e Tecnologia, Universidade Nova de Lisboa, 2829-516 Almada, Portugal, and also with UNINOVA, 2829-517 Costa da Caparica, Portugal (e-mail: pmc@uninova.pt).

Digital Object Identifier 10.1109/LCOMM.2016.2598334 guaranteed, especially, when larger modulation indexes are considered. On the other hand, it was recently shown that the nonlinear distortion effects can be used to improve the performance of OFDM schemes, provided that an optimum detection is considered [6], [7]. The optimum detection of CE-OFDM schemes in ideal additive white Gaussian noise (AWGN) channels was analyzed in [5] and it was shown that CE-OFDM can even outperform the conventional, linear OFDM.

In this letter, we study the optimum performance of CE-OFDM in both ideal AWGN and frequency-selective channels. Moreover, we compare the optimum detection performance with the performance of conventional CE-OFDM receivers that involve a phase detector. It is shown that the phase detector can achieve the optimum performance in ideal AWGN, but only for small modulation indexes, where the power efficiency is very low. For this reason, the optimumbased detection of CE-OFDM signals seems to be desirable, especially for scenarios with severe power constraints.

\section{CE-OFDM ChARACTERIZATION}

Each CE-OFDM symbol is composed by $N$ complex data symbols that form the block $\mathbf{S}=\left[\begin{array}{llll}S_{0} S_{1} & \ldots & S_{N-1}\end{array}\right]^{T}$. To guarantee that the phase modulator's input is real-valued, the data symbols are constrained to have Hermitian symmetry, which means that $S_{k}=0$ for $k \in 0, N / 2$ and $S_{N-k}=S_{k}^{*}$, otherwise. The data symbols are selected from a quadrature phase shift keying (QPSK) constellation and are of the form $S_{k}= \pm A \pm j A$. To obtain an oversampled version of the CE-OFDM signal, the data block is augmented with $N(M-1)$ idle subcarriers, i.e., with $S_{k}=0$, where $M$ denotes the oversampling factor. In these conditions, the inverse discrete Fourier transform (IDFT) yields the block of time-domain samples $\mathbf{s}=\left[\begin{array}{lll}s_{0} s_{1} & \ldots & s_{N M-1}\end{array}\right]^{T}$. The $n$th sample of this block has a Gaussian distribution with zero mean and variance $\mathbb{E}\left[\left|s_{n}\right|^{2}\right]=\sigma^{2}=2 A^{2}(N-2) /(N M)^{2}$. These time-domain samples are submitted to a phase modulator, whose the output for the $n$th time-domain sample is [5]

$$
y_{n}=f\left(s_{n}\right)=\exp \left(j 2 \pi h s_{n} / \sigma\right),
$$

where $f(\cdot)$ is the phase modulation function and $h$ represents the modulation index. For ideal AWGN channels, the received signal is

$$
z_{n}=y_{n}+v_{n}=\exp \left(j 2 \pi h s_{n} / \sigma\right)+v_{n},
$$

where $v_{n}$ represents the $n$th AWGN sample and $\mathbb{E}\left[\left|v_{n}\right|^{2}\right]=$ $N_{0}$ is the one-sided noise power spectral density (PSD). Conventionally, the detection procedure of CE-OFDM involves a phase detector followed by a discrete Fourier transform (DFT) [3]. By considering the power series approximation for the complex exponential in (2), as well as low 